\title{
Le romantisme de Consuelo, ou la méditation sur les Lumières de George Sand
}

\section{Damien Zanone}

\section{(2) OpenEdition}

Journals

Édition électronique

URL : http://journals.openedition.org/rief/254

DOI : $10.4000 /$ rief.254

ISSN : 2240-7456

Éditeur

Seminario di filologia francese

Référence électronique

Damien Zanone, «Le romantisme de Consuelo, ou la méditation sur les Lumières de George Sand ", Revue italienne d'études françaises [En ligne], 3 | 2013, mis en ligne le 15 décembre 2013, consulté le 05 mai 2019. URL : http://journals.openedition.org/rief/254; DOI : 10.4000/rief.254

Ce document a été généré automatiquement le 5 mai 2019.

\section{(c) (i) (9)}

Les contenus de la RIEF sont mis à disposition selon les termes de la Licence Creative Commons Attribution - Pas d'Utilisation Commerciale - Pas de Modification 4.0 International. 


\title{
Le romantisme de Consuelo, ou la méditation sur les Lumières de George Sand
}

\author{
Damien Zanone
}

1 George Sand est probablement, parmi les écrivains de sa génération, l'un de ceux qui montrent l'imaginaire dix-huitiémiste le plus étendu et le plus subtil. Un détour par Histoire de ma vie, publiée douze ans après Consuelo, permet d'en faire l'archéologie en constatant que le premier relais fut la mémoire vivante de la grand-mère, fille du maréchal de Saxe et, par là, apparentée à l'aristocratie germanique qu'évoque Consuelo. Dans l'autobiographie, le retour sur la vie de cette grand-mère, née Aurore de Saxe, amène à rencontrer des noms dont le roman, quelques années plus tôt, a déjà fait usage : le baron de Trenck, la princesse Amélie de Prusse (sœur de Frédéric II), la Dauphine de France (fille du roi de Saxe). La médiation livresque ensuite, en particulier la lecture de Mémoires du XVIII ${ }^{\mathrm{e}}$ siècle qui figurent parmi les sources documentaires de l'écrivain, a servi à préciser des faits plus qu’à cerner des mœurs déjà connues par tradition familiale.

2 Consuelo et La Comtesse de Rudolstadt constituent une vaste fresque qui reflète, sur le plan éditorial, des circonstances nouvelles propres aux années 1840 : le roman est d'abord publié en feuilleton pendant deux ans (1842-1844) avant de l'être en volume. De l'aveu de l'auteur qui revient sur cette expérience dans une préface de 1855, ce fut l'épreuve d'une "publication pour ainsi dire forcée » puisqu'elle obligeait la marche de l'intrigue à respecter les éléments déjà connus du public et sur lesquels il n'était pas possible de revenir : de là, un roman qui «va souvent un peu à l'aventure ", à la suite de son héroïne poussée sur les routes ${ }^{1}$. Le récit commence comme une nouvelle centrée, à Venise, sur la musique et la vie des théâtres; il bascule dans un long développement de modèle gothique (dans un château de Bohême dont l'exploration permet de convoquer plusieurs siècles de l'histoire de ce pays); il s'ouvre ensuite à une matière historique contemporaine à son action, avec la représentation de la cour de Vienne et de celle de Berlin au temps des règnes de Marie-Thérèse et de Frédéric II; il s'achève enfin, en 
dehors de tout espace nettement défini, dans un envol de fabulation mystique, l'hérö̈ne ayant rejoint la société secrète des «Invisibles » pour laquelle elle continue son " ardent et infatigable pèlerinage à travers la France, l'Espagne, l'Angleterre et l'Italie $»^{2}$.

3 L'Europe et le XVIII ${ }^{e}$ siècle sont donc le vaste cadre de ce roman. Dans le dernier chapitre, peu avant un long épilogue qui vient, à vrai dire, ajouter autant de trouble que de sens, une formidable péroraison se développe, directement énoncée par la voix narrative, pour déclarer que faire le portrait du XVIII ${ }^{e}$ siècle avec le trop-plein de ses éléments contraires était un des enjeux du roman :

Un tel contraste est un des traits les plus saisissants de ce dix-huitième siècle, trop rempli d'idées et de travail intellectuel de tous les genres, pour que la synthèse ait pu en être déjà faite avec clarté et profit par les historiens philosophiques de nos jours. [...] Mais peu à peu la lumière sortira de ce chaos; et si notre siècle arrive à se résumer lui-même, il résumera aussi la vie de son père le dix-huitième siècle, ce logogriphe immense, cette brillante nébuleuse, [...] laboratoire effrayant, où tant de formes hétérogènes ont été jetées dans le creuset, qu'elles ont vomi, dans leur monstrueuse ébullition, un torrent de fumée où nous marchons encore enveloppés de ténèbres et d'images confuses. ${ }^{3}$

4 «Logogriphe immense »... Le dictionnaire nous dit, de ce jeu d'esprit qui consiste à reconnaître un mot à partir d'autres mots qui en utilisent les lettres, qu'il a donné son nom pour désigner un propos énigmatique ou, si l'on prend la chose en mauvaise part, obscur, confus, incohérent. Or qui sont, au XIX siècle, « les historiens philosophiques de nos jours » à même de déchiffrer un tel logogriphe, si ce ne sont les romanciers? Sand, sans doute, l'a déclaré moins ouvertement que Balzac (elle a d'ailleurs renoncé à préfacer La Comédie humaine, ce qui a laissé à Balzac l'occasion de perpétrer l'annexion de l'histoire par le roman dans le célèbre "Avant-propos») mais n'en pense pas moins. Voici comment, dans la préface rédigée dix ans après la publication de son roman, Sand explique le brusque élargissement des ambitions narratives de celui-ci: "J'avais commencé Consuelo avec le projet de ne faire qu'une nouvelle. Ce commencement plut, et on m'engagea à le développer, en me faisant pressentir tout ce que le dix-huitième siècle offrait d'intérêt sous le rapport de l'art, de la philosophie et du merveilleux $»^{4}$. De ces trois aspects, le roman met surtout en valeur le premier et le troisième : ce dernier, le merveilleux, étant celui que la postérité retient le moins volontiers du XVIII ${ }^{\mathrm{e}}$ siècle, c'est un de ses mérites de le faire valoir en convoquant la mémoire d'un siècle de l'illuminisme plutôt que des Lumières. Le fait est que celles-ci sont laissées pour parent pauvre ; que la philosophie ait été déclarée objet d'intérêt à cultiver n'y change rien. Dans le roman comme dans la préface déjà citée, le nom de Voltaire ne se rencontre que dans le voisinage de celui de Cagliostro pour définir l'esprit du siècle. Le roman se met à la trace de ce «siècle étrange, qui commence par des chansons, se développe dans des conspirations bizarres, et aboutit, par des idées profondes, à des révolutions formidables $! »^{5}$ Encadré par ces propos tenus dans la préface et le dernier chapitre, le roman voit donc son programme clairement établi : il est contemplation et tentative de déchiffrement du logogriphe, il est une méditation sur lui.

Le dernier tiers du roman, publié sous le titre de La Comtesse de Rudolstadt, en est la partie herméneutique dans laquelle on observe au mieux cette méditation. La Comtesse de Rudolstadt narre le séjour de l'héroïne à la cour de Prusse, ses démêlés avec Frédéric II, son enfermement dans la forteresse de Spandaw, son évasion favorisée par la société secrète des Invisibles, ordre auquel elle est initiée et s'attache désormais, avant que le roman ne s'achève comme « un beau fleuve » qui « se ramifie vers son embouchure et se 
perd en mille filets capricieux dans les sables dorés de la grève $»^{6}$. Même si l'intrigue de ce dernier tiers de Consuelo reste marquée par une action soutenue, La Comtesse de Rudolstadt se signale particulièrement comme une vaste séquence méditative où, par un système de récits enchâssés, la matière narrative déjà accomplie dans les deux premiers tiers est reprise, racontée à nouveau selon des points de vue de personnages pour être réfléchie encore et pour mieux trouver sa leçon. La narration de La Comtesse de Rudolstadt semble moins motivée par la volonté de raconter encore du nouveau, d'aller à l'aventure, que de tirer les leçons de cette aventure pour publier les fins dernières de tout ce qui, du « logogriphe immense », aura été décrit morceau par morceau jusque là. Or, La Comtesse de Rudolstadt nous parle-t-elle des Lumières?

6 La philosophie du XVIII ${ }^{e}$ siècle, même si Sand préfacière la désigne au nombre des intérêts qui occupe le roman, n'y apparaît guère et, en tout cas, pas sous l'aspect rayonnant et institué des Lumières (le terme, d'ailleurs, n'est pas employé en ce sens). Cela advient cependant, dans les premiers chapitres de La Comtesse de Rudolstadt dont la scène est à la cour de Frédéric II, et le traitement en est satirique. Deux aspects sont significatifs à cet égard.

7 Tout d'abord, la représentation bouffonne qui est donnée des Lumières françaises à travers une longue scène de souper dont les convives sont, outre Frédéric II, l'élite intellectuelle que celui-ci a rassemblée (Voltaire, La Mettrie, le marquis d'Argens, le surnommé Quintus Icilius et Francesco Algarotti). Ce souper ne sait ce qu'il doit être d'abord, d'une réunion d'esprits libres ou de courtisans timorés. L'un des convives, La Mettrie, opte sans réserve pour le rôle d'esprit libre, ce qui lui vaut d'être la figure de proue de cette assemblée : il est le seul à «prendre au pied de la lettre le philosophisme égalitaire que le roi affectait dans sa vie intime » et la règle d'étiquette que ce roi avait posée «"Il n'y a pas de roi quand on soupe." »" La Mettrie dialogue avec le roi quand les autres ne font que lui répondre en propos mesurés et, dans ce foyer des Lumières qui est circonscrit en Prusse au logis du roi, il occupe le premier rôle: mais ce rôle est exactement celui de bouffon du roi ! Tous ses propos sont émaillés d'audace vis-à-vis de Frédéric II : parole très libre qui choque les autres, ton ironique pour lui donner du " Votre Majesté ». Ses discours désordonnés obéissent à ses humeurs plus qu'à la raison. Il s'en prend d'ailleurs plaisamment à celle-ci dans une tirade vive et provocante, suscitée par une phrase de Frédéric ( $\mathrm{Oh}$ ! la raison, mon cher Voltaire, que son règne nous arrive !») dont il prend le contrepied : «La raison, la raison! dit La Mettrie, je la trouve séante et bénévole quand elle me sert à excuser et à légitimer mes passions, mes vices... ou mes appétits... [...] mais quand elle m'ennuie, je demande à être libre de la mettre à la porte. [...] Que son règne n'arrive pas $!^{8}$

8 Le premier rôle n'est donc pas donné, dans cette assemblée, à celui qu'on pourrait croire : «le grand Voltaire boudait la France» mais «commençait à se désillusionner singulièrement de la Prusse ". Celui dont la qualité de "premier chambellan " est rappelée, ainsi que le montant des appointements qu'elle lui vaut, fait acte de présence laborieuse, en proie à « des douleurs d'entrailles » et à « ce spleen prussien qui s'emparait bien vite de tous les heureux mortels appelés à contempler Frédéric dans sa gloire $»^{10}$. Les interventions orales du personnage Voltaire se limitent donc à commenter les bons mots du personnage La Mettrie et à adresser, comme par devoir mécanique, des propos flagorneurs au roi. La seule fois où le texte donne accès à son for intérieur, c'est pour l'entendre s'ennuyer du souper bouffon et se dire « j'ai la colique » ${ }^{11}$. 
9 L'autre aspect du traitement satirique des Lumières est plus large, dépasse la scène particulière du souper entre philosophes patentés pour orienter dans son entier la représentation de la cour de Frédéric II et aboutir à cette pensée de Consuelo qui en fait le résumé : « il y a décidément, dans ce royaume de la raison, une conspiration permanente contre la raison $»^{12}$. Ce qui justifie l'énoncé n'est pas tant le désordre toléré de La Mettrie (somme toute, des discours déviants tenus devant un petit nombre) que l'emprise généralisée de la superstition. "Prédire l'avenir », « révéler les trésors cachés » et « faire de l'or » sont les « talents de société ${ }^{13}$ généralement pratiqués dans le palais de Frédéric et de sa cour: divers magiciens (et non des moindres: le comte de Saint-Germain, Cagliostro, ainsi que, comme créature propre à cette fiction, le dénommé Trismégiste) y trouvent occasion d'avoir dans le château une place marquée et une vie sociale privilégiée. Savoir s'il convient de les qualifier de charlatans n'est pas une question réglée mais fait l'objet de discussions animées. Les agissements de Cagliostro, en particulier, entrent dans l'intrigue et constituent un fil de tension narrative : le roi-philosophe luimême, qui pourtant est le premier à déplorer qu'en son pays le peuple honore plus Cagliostro que Voltaire ${ }^{14}$, narre à ses convives, lors du souper philosophico-bouffon, l'apparition d'un revenant que Cagliostro serait parvenu à susciter aux yeux de la sage Consuelo. Dans le palais de la raison dont il est censé être le garant, il articule donc luimême le merveilleux.

La première page de la longue séquence du roman située dans ce palais donne d'emblée le la. L'expression musicale convient pour parler de ce qui est alors montré: une représentation d'opéra qui s'interrompt sur un malaise de la cantatrice, Consuelo, arrêtée en plein milieu d'un air par on ne sait quelle vision (on apprendra par la suite qu'elle a vu, dans l'assistance, son époux pourtant mort) : « 0 mon Dieu! $»^{15}$ s'écrie-t-elle, premières paroles que le roman fait résonner chez le roi-philosophe... À partir de là, dans l'expérience qu'en fait l'héroïne et, à travers elle, le lecteur, le château de ce roi n'est plus qu'un nouveau "château de la subversion ", qu'il convient de désigner par l'expression promue par Annie Le Brun pour désigner l'imaginaire gothique ${ }^{16}$. Ce château où l'on se perd entre "profondes galeries " et "mystérieux escaliers", où l'on craint la nuit de croiser « la Balayeuse », où l'on s'échange des lettres chiffrées en signes cabalistiques ${ }^{17}$, n'a pas grand chose à envier au "Château des Géants " perdu au fond de la forêt de Bohême et dont l'évocation, beaucoup plus tôt dans le roman, s'était faite en référence déclarée aux romans d'Ann Radcliffe. Petites ou grandes choses : rien donc n'est gouverné selon la raison et le roi en est le premier instigateur. Il est surpris, dans une scène de dispute avec Consuelo, à menacer de la frapper avec sa canne avant de finalement jeter celle-ci au feu. Cette canne, apprend-on, était un cadeau reçu de Voltaire : loin de l'aider à marcher, elle était devenue «canne-sceptre " entre les mains d'un tyran ${ }^{18}$. Le rassurant constat que fait un personnage trop naïf en évoquant « le brillant siècle de raison où nous avons le bonheur de vivre $»^{19}$ finit donc par sonner comme une antiphrase : ce n'est certes pas dans le palais du roi-philosophe qu'on en fait l'expérience !

11 La satire étant faite, les Lumières ne rayonnent plus: elles ne peuvent plus prétendre porter le sens du siècle auquel elles ont réussi à associer leur nom. Ce n'est pas avec elles qu'on déchiffrera le «logogriphe immense »: mais alors avec quoi? Ou, pour poser la question qu'emploie le roman à l'approche de sa fin, d'où vient le «courant électrique d'enthousiasme sublime, de foi ardente et de fanatisme terrible» qui a mis en mouvement la Révolution française ${ }^{20}$ ? 
12 C'est du côté de l'illuminisme que le roman va le chercher, parmi les «cerveaux contemplatifs de la patiente Germanie $»^{21}$ que la narration met en perspective les uns par rapport aux autres: Jacques Bœhm en particulier, mystique du début du XVII ${ }^{\mathrm{e}}$ siècle, tient le haut bout de la chaîne que finit Swedenborg ${ }^{22}$. Le courant illuministe - et notamment la secte bavaroise qui se désigne ainsi dans les années 1770 - est donné pour un mouvement continué sur plusieurs siècles, depuis la révolte des hussites dans la Bohême du $\mathrm{XV}^{\mathrm{e}}$ siècle et jusqu'à la Révolution française : toujours il aurait approfondi le lien entre interrogation religieuse et contestation sociale. Cette transmission au long cours a été rendue possible par les sociétés secrètes: celle qu'invente le roman, les "Invisibles », prend place dans une longue lignée. Cette secte qui énonce en trois mots « le secret de [ses] mystères" ( Liberté, fraternité, égalité » ${ }^{23}$ ) n'est pas la moindre des merveilles du XVIII ${ }^{\mathrm{e}}$ siècle de Consuelo :

Bien qu'un siècle à peine nous sépare de l'existence des Invisibles, elle est problématique pour l'historien; mais trente ans plus tard l'illuminisme reprit ces formes ignorées du vulgaire, et, puisant à la fois dans le génie inventif de ses chefs et dans la tradition des sociétés secrètes de la mystique Allemagne, il épouvanta le monde [...]. Un demi-siècle avant ces jours marqués par le destin, tandis que la monarchie galante de Louis XV, le despotisme philosophique de Frédéric II, la royauté sceptique et railleuse de Voltaire, [...] semblaient n'annoncer pour longtemps au monde que décrépitude, antagonisme, chaos et dissolution, la Révolution française fermentait à l'ombre et germait sous terre. ${ }^{24}$

George Sand, qui est en ses belles années socialistes au moment où elle écrit Consuelo, a déjà dit deux ans auparavant, en préface à son roman Le Compagnon du tour de France (1840) tout le bien qu'elle pensait des sociétés secrètes comme lieux d'élaboration théorique du sens de l'histoire et de premier accomplissement concret de ce sens à travers des actions anticipatrices de l'avenir. Il lui plaît de reprendre à son compte, pour la retourner, la théorie du complot mise au point, dès après la Révolution, par des auteurs contre-révolutionnaires pour expliquer celle-ci (par exemple l'abbé Barruel dans les Mémoires pour servir à l'histoire du jacobinisme, 1797). Le roman évoque ainsi, par la voix de divers de ses personnages, l'importance décisive qu'ont eue différentes sectes à plusieurs époque de l'histoire ${ }^{25}$.

Cependant, au delà de l'illuminisme comme courant spécifique ayant eu ses références propres à un moment du XVIII siècle, ce qui semble en jeu et objet de quête à travers la méditation sur le logogriphe, c'est l'idéal, entendu comme reformulation dynamique des Lumières: celles-ci ne sont plus pensées comme un ensemble acquis de principes à préserver mais comme un mouvement pour faire advenir ces principes dans le réel, pour faire que le réel soit enfin le lieu d'une conjonction trouvée entre les faits et les principes, entre le visible et l'invisible. Travailler à la rencontre des deux est vraiment l'œuvre propre du romantisme.

La méditation sur les Lumières de George Sand, dans Consuelo, s'arrache donc aux contingences historiques pour devenir une méditation sur l'idéal. La partie italienne du roman explore la façon dont, dans les arts - et dans le chant en particulier - le plaisir sensuel peut être mis au service d'une expression spirituelle. La partie germanique, ensuite, procède à un examen tourmenté des états de société pour voir dans quelle mesure ils sont conciliables avec une réforme qui les rapproche du message égalitaire d'abord porté par les Évangiles.

George Sand écrit donc, avec Consuelo, à la fois son De l'Italie et son De l'Allemagne. Comme Germaine de Staël avant elle dans Corinne ou l'Italie et comme Voltaire dans le texte même 
de Consuelo, Sand semble bouder la France avec ce roman: comme Corinne, Consuelo emprunte des chemins qui lui font contourner la France et accomplir un parcours symbolique en terres d'émigration. Faut-il en déduire que la logique de ces romans de l'artiste est de mener une quête de l'idéal dans le déni du réel ? On reconnaîtrait plus justement leur apport en établissant que leur tension inquiète vers l'idéal est une reformulation des Lumières propre à l'usage que le $\mathrm{XIX}^{\mathrm{e}}$ siècle peut en faire, c'est-à-dire après que le réel a fait enregistrer un certain nombre de déceptions.

\section{NOTES}

1. G. Sand, Consuelo. La Comtesse de Rudolstadt, éd. D. Zanone, Paris, Robert Laffont, « Bouquins », 2004, p. 29.

2. Ibid., p. 1115. La dimension européenne de cette mission est déjà indiquée p. 1107.

3. Ibid., p. 1090-1091.

4. Ibid., p. 30.

5. Ibid., p. 31.

6. Ibid., p. 1110.

7. Ibid., p. 747 et p. 751.

8. Ibid., p. 754.

9. Ibid., p. 738-739.

10. Ibid., p. 739 et p. 756 .

11. Ibid., p. 757.

12. Ibid., p. 828.

13. Ibid., p. 783.

14. Ibid., p. 755 .

15. Ibid., p. 740.

16. A. Le Brun, Les Châteaux de la subversion, Paris, Gallimard, « Tel », 2010 [1982].

17. G. Sand, Consuelo. La Comtesse de Rudolstadt, op. cit., p. 747, p. 822 et p. 765.

18. Ibid., p. 834.

19. Ibid., p. 781.

20. Ibid., p. 1090.

21. Ibid., p. 911.

22. Ibid., p. 910 et p. 915.

23. Ibid., p. 1010.

24. Ibid., p. 1090.

25. Ibid., p. 792, p. 912, p. 914, p. 1007-1008. 
INDEX

Mots-clés : Lumières, romantisme, Sand (George), Consuelo, Comtesse de Rudolstadt, satire, La Mettrie (Julien Offray de) 